\title{
Transmission priority scheme with adaptive backoff technique in fiber-wireless networks
}

\author{
Wan Hafiza Wan Hassan ${ }^{1,2 *}$, Horace King ${ }^{1}$, Shabbir Ahmed ${ }^{1}$ and Mike Faulkner ${ }^{1}$
}

\begin{abstract}
This paper considers the realization of fiber-wireless (Fi-Wi) networks using the gigabits passive optical network (GPON) and the infrastructure-based wireless local area network (WLAN). The bottleneck of such hybrid system is the WLAN where interference limits the performance. As such, we focus on enhancing the WLAN performance by analytically deriving the optimum contention window (CW) sizes of access points (APs) and wireless users (WUs), respectively. An adjustable transmission priority factor is introduced to allow uplink-downlink transmission fairness. Further, an adaptive backoff technique using information from monitoring the GPON and WLAN networks is proposed. Simulations show the CW sizes of all WUs are maintained within a standard deviation of $1.5 \%$ at a cost of a $3 \%$ loss in throughput due to the effect of convergence and other estimates.
\end{abstract}

Keywords: Fi-Wi networks; WLAN; GPON; Contention window (CW); Adaptive backoff; Access point (AP); Wireless user (WU)

\section{Introduction}

Fiber-wireless (Fi-Wi) networks combine the capacity of optical fiber networks with the ubiquity and mobility of wireless networks [1-7]. The use of energy-efficient and stable passive optical splitters makes them the favored choice among other fiber-to-the-home (FTTH) technologies. Gigabits passive optical network (GPON) and Ethernet passive optical network (EPON) are the major standards in PONs. In comparison to EPON, the current GPON offers higher capacity per user and allows more users per PON [8]. This makes GPON more attractive, and hence, it is adopted by major telecommunications service providers (e.g., National Broadband Network (NBN) in Australia and Ultra-Fast Broadband (UFB) network in New Zealand [9]).

In this work, we focus on the integration of GPON with a 'closed' WLAN system as shown in Figure 1. In this situation, the operator has a dedicated spectrum allocation which is shared by all access points. The integration is employed at the end-user terminal where an optical network terminal (ONT) of GPON and an access point (AP)

\footnotetext{
*Correspondence: wanhafiza.wanhassan@live.vu.edu.au

${ }^{1}$ College of Engineering and Science, Victoria University, Ballarat Road, 8001 Melbourne, Australia

${ }^{2}$ School of Ocean Engineering, Universiti Malaysia Terengganu 21030 Kuala Terengganu, Malaysia
}

of WLAN are incorporated into a single device, forming a Fi-Wi hybrid system (also known as radio and fiber (R\&F) system) $[3,10]$. The integrated ONT-AP device acts as a gateway translating media access control (MAC) frames from the optical network to the wireless network and vice versa. Two distinct separate MAC protocols are used to access GPON and WLAN, respectively. Thus, the wireless MAC frames only traverse the WLAN and do not have to travel along the optical fiber to be processed at the central office. In contrast, radio over fiber (RoF), another possible enabling technology for the Fi-Wi system, transmits radio frequency (RF) signals from the central office to the remote access units (RAUs) as illustrated in Figure 2. The central office in RoF controls both optical and wireless media compared to just optical in R\&F. This difference makes R\&F more practical compared to RoF because an optical distribution use of wireless signals imposes additional fiber propagation delays which might exceed certain wireless MAC protocol timeouts and further worsens the overall network performance $[10,11]$.

However, the increasing number of wireless terminals within households, especially in dense urban and suburban areas, results in dominant interference or congestion issues in WLANs. Such congestions occur when a WLAN channel is shared by many users. Hence, the scope of this paper is fully focused on the WLAN performance

\section{Springer}

(c) 2015 Wan Hassan et al.; licensee Springer. This is an Open Access article distributed under the terms of the Creative Commons Attribution License (http://creativecommons.org/licenses/by/4.0), which permits unrestricted use, distribution, and reproduction in any medium, provided the original work is properly credited. 


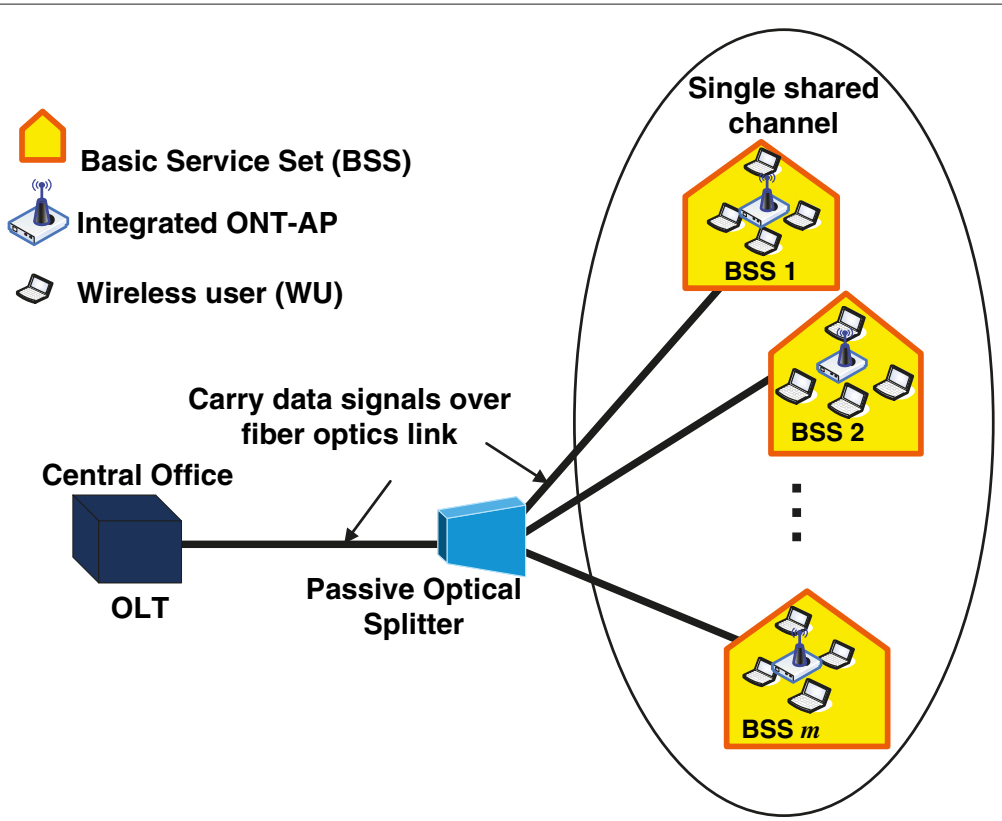

Figure 1 The Fi-Wi network scenario using the R\&F technology.

where multiple APs are connected through GPON at close proximity to each other and share one single channel to serve their respective wireless users (WUs).

WLAN adopts the IEEE 802.11 standard which specifies the distributed coordination function (DCF) as its main medium access control protocol. DCF is a random access scheme, based on the carrier sense multiple access with collision avoidance (CSMA/CA) [12]. This protocol only allows a station to initiate a transmission after it senses the channel is unoccupied for a period defined as DCF interframe space (DIFS). If the channel is sensed busy either immediately or within the DIFS period, the station keeps on listening on the channel until it is sensed idle for a DIFS period. Consequently, the station generates a backoff interval which is randomly chosen from the backoff window (known as contention window $(\mathrm{CW})$ size)

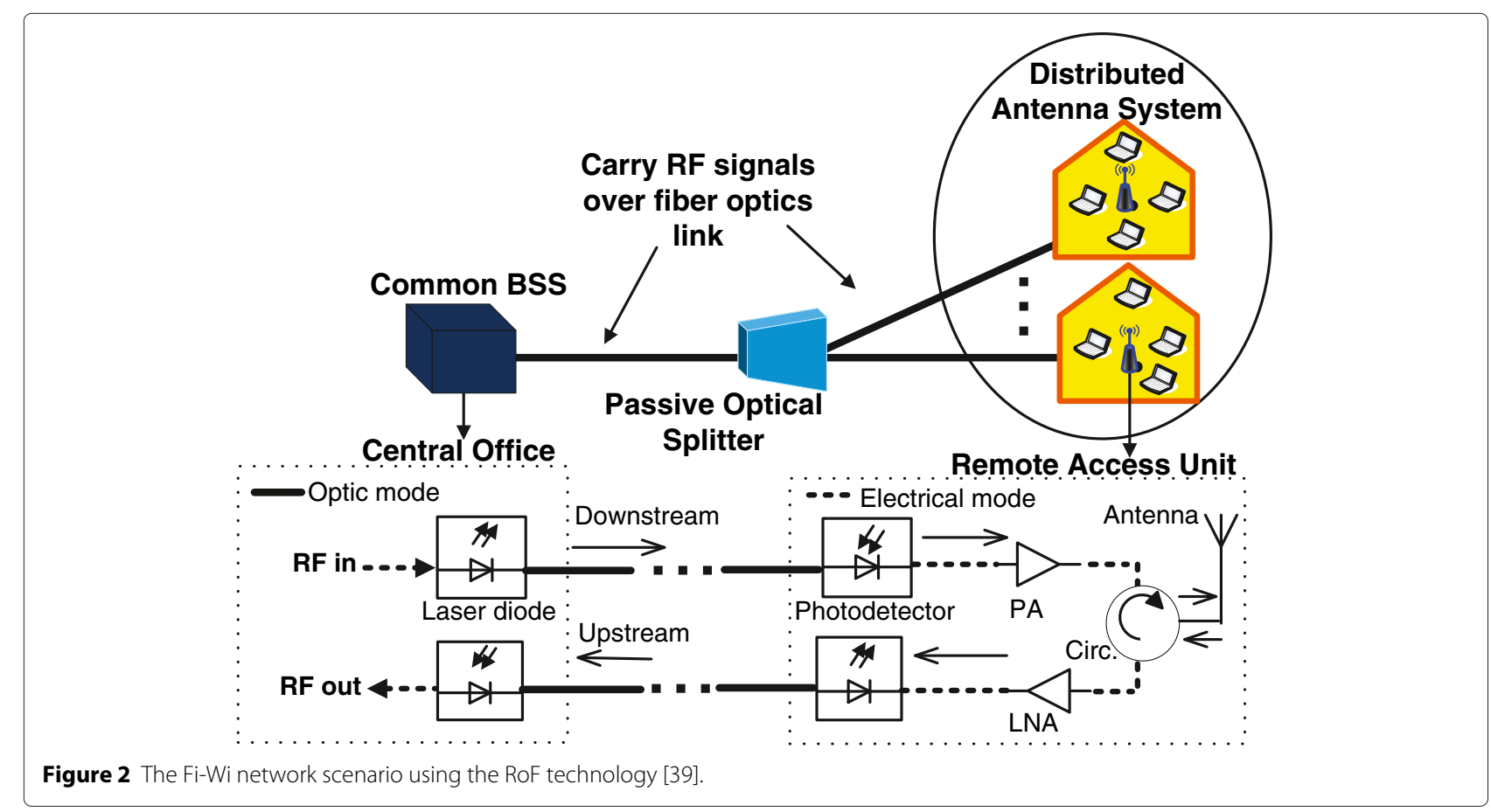


[13]. The window size begins with a minimum CW size, and it is doubled at each retransmission up to a maximum CW size. Retransmission takes place whenever there is a packet collision, indicated by the absence of acknowledgment frame (ACK) from the receiver. This backoff algorithm is known as binary exponential backoff (BEB) technique [12].

It is extensively agreed that the $\mathrm{BEB}$ algorithm adopted in the MAC protocol for the IEEE 802.11 standard is the key factor to the WLAN performance degradation [13-22]. There are two major drawbacks found in BEB that cause the network degradation as discussed in [17]. First, the contention window is increased upon transmission failure regardless of the cause of failure. Second, after a successful packet transmission, the contention window is reset to the minimum size, thus forgetting its knowledge of the current congestion level in the network and increasing its collision chances.

Numerous modifications have been proposed in literature to improve the legacy BEB algorithm. In [14], the authors proposed to exponentially increase the CW size when there is a collision and exponentially decrease the CW size when there is a successful transmission, known as the EIED (exponentially increases exponentially decreases) algorithm. The exponential factors are then optimized to get maximum throughput. The proponents of [15] use a more conservative approach; they linearly decrease the CW size when there is a successful transmission. Similarly, [20] proposes to halve the CW size (unlike BEB where the CW size is reset to minimum) to increase the overall throughput. The IEEE 802.11e standard changes the minimum and maximum $\mathrm{CW}$ limits in the $\mathrm{BEB}$ algorithm to define different quality of service access categories [23,24]. In common, all of the above techniques steadily decrease the CW after a successful transmission to retain the overall network state information. Though this approach may have a positive correlation, it usually falls short of predicting the overall network status.

Previous works in [18] and [19] have shown that if exact knowledge (e.g., number of active stations and packet size) of the network is known, the CW size can be tuned to achieve a protocol capacity very close to its theoretical bound. In reality, it is almost impossible for stations to obtain an exact knowledge of the network, only estimates are likely. Listening to the channel to obtain the average packet length and slot transmission probability (alternatively described as slot utility or number of free slots) is the usual starting point [25].

The adaptive window algorithm (AWA) proposed by Bianchi et al. [22] used the number of active stations in the network to control the optimum CW size. A similar approach is employed in [18] and [19] to estimate the number of active stations which is then used in a complex $p$-persistent MAC protocol to select the optimum backoff interval. In some cases, it is possible to avoid the intermediate step of estimating the number of users $[21,25,26]$.

In common, the above studies focused on the enhancement of the IEEE 802.11 protocol with all stations having an equal chance to transmit. In infrastructure networks, the AP requires more transmission opportunities to give fairness to the uplink/downlink performance [27]. Priority access for an individual user can be obtained by scaling the CW [26], changing the $C W_{\min }$ [28-32], utilizing reduced inter-frame spacings (PIFS and SIFS instead of DIFS) $[33,34]$, and adjusting transmission opportunity (TXOP) limits $[31,35]$. Such techniques have been proposed for QoS enhancements and to provide uplink/downlink fairness in a single BSS infrastructure network. They are less suitable for an infrastructure WLAN network where there is more than one AP sharing the same spectrum and each serving its own basic service set (BSS) of associated WUs.

In this paper, we employ a novel approach known as transmission priority (TxPriority) scheme by introducing a priority factor that can be adjusted to allow AP transmission priorities as required within an infrastructure WLAN network. Mathematical analysis is carried out, and expressions are derived for optimum AP and WU contention window sizes. The derived formulations show that the optimum CW sizes depend on the number of active APs and WUs; unknown quantities. We capitalize on information from both GPON and WLAN networks to provide appropriate estimates and make the system adaptive. A new convergence function is introduced to improve the reliability of the adaptive system. Our mathematical formulations are verified using network simulator software OPNET 16.1 [36].

The remaining sections of this paper are organized as follows: 'Transmission priority scheme' section describes the transmission priority scheme and derives optimum contention window sizes. 'GPON network indicator' section outlines the GPON architecture and defines an indicator to estimate the number of active APs. 'Adaptive backoff technique' section estimates the number of active WUs and proposes an adaptive system. 'The convergence function' section presents a new convergence function. Finally, 'Conclusions' section concludes the paper and provides directions for future work.

\section{Transmission priority scheme}

This section derives optimum contention window sizes for the two types of contending stations, an AP and a WU in the WLAN network. The analysis considers the worst case scenario in terms of frequency availability in densely populated urban areas. All BSSs are within close proximity, i.e., all wireless stations, APs and WUs, share one single channel and can hear each other, albeit being 
potential interferers. The spectrum is considered 'closed', that is a single $20-\mathrm{MHz}$ channel entirely dedicated to this Fi-Wi network. When a station wants to contend the channel, the backoff counter is randomly selected within the range $(0, C W-1)$ and then decremented at each subsequent idle slot.

Consider a Fi-Wi network scenario comprising of $m$ integrated ONT-APs and $n$ associate WUs, contending the WLAN channel with contention window sizes of $C W_{\text {ap }}$ and $C W_{\text {wu }}$, respectively. The probability that there is at least one transmission in a slot is given by,

$$
P_{\mathrm{tr}}=1-\left(1-p_{\mathrm{ap}}\right)^{m}\left(1-p_{\mathrm{wu}}\right)^{n}
$$

where $p_{\mathrm{ap}}$ and $p_{\mathrm{wu}}$ are the probability of an AP and a WU transmitting. See Table 1 for a list of notations. The probabilities $p_{\text {ap }}$ and $p_{\text {wu }}$ are independent to each other and are given by the following, based on Bianchi's model in [22],

$$
p_{\text {ap }}=\frac{2}{\left(\mathrm{CW}_{\mathrm{ap}}+1\right)}
$$

and,

$$
p_{\mathrm{wu}}=\frac{2}{\left(\mathrm{CW}_{\mathrm{wu}}+1\right)} .
$$

Further, the average number of consecutive idle slots per transmission $E[I]$ can be defined as

$$
E[I]=\frac{1-P_{\mathrm{tr}}}{P_{\operatorname{tr}}}
$$

The probability that a transmission is a successful AP transmission $P_{s}^{\text {ap }}$ is given by the probability that only one AP transmits on the channel provided that there is a transmission,

$$
P_{s}^{\mathrm{ap}}=\frac{m p_{\mathrm{ap}}\left(1-p_{\mathrm{ap}}\right)^{m-1}\left(1-p_{\mathrm{wu}}\right)^{n}}{P_{\mathrm{tr}}}
$$

\section{Table 1 List of notations}

\begin{tabular}{ll}
\hline Notation & Description \\
\hline$p_{\text {ap }}$ & Probability of an AP transmitting \\
\hline$p_{\text {wu }}$ & Probability of a WU transmitting \\
\hline$m$ & Total number of APs (or BSS's) \\
\hline$n$ & Total number of WUs \\
\hline$C W_{a p}$ & Current contention window size for an AP \\
\hline$C W_{w u}$ & Current contention window size for a WU \\
\hline$P_{s}^{\text {ap }}$ & Probability that a transmission is a successful AP \\
\hline$P_{s}^{\text {wu }}$ & transmission \\
\hline & Probability that a transmission is a successful WU \\
& transmission
\end{tabular}

Similarly, the probability that a transmission is a successful WU transmission is given by

$$
P_{s}^{\mathrm{wu}}=\frac{n p_{\mathrm{wu}}\left(1-p_{\mathrm{ap}}\right)^{m}\left(1-p_{\mathrm{wu}}\right)^{n-1}}{P_{\mathrm{tr}}} .
$$

A transmission priority factor $k$ can now be introduced. It is defined as the number of successful transmissions of WUs with respect to APs,

$$
k=\frac{P_{s}^{\mathrm{wu}}}{P_{s}^{\mathrm{ap}}}=\frac{n p_{\mathrm{wu}}\left(1-p_{\mathrm{ap}}\right)}{m p_{\mathrm{ap}}\left(1-p_{\mathrm{wu}}\right)} .
$$

A value of $k<1$ implies higher downlink throughput; while a value of $k>1$ represents higher uplink throughput.

Note, successful transmissions from an AP and a WU are mutually exclusive events. Thus, the probability that a transmission is successful is given by

$$
P_{s}=P_{s}^{\mathrm{ap}}+P_{s}^{\mathrm{wu}}=(1+k) P_{s}^{\mathrm{ap}} .
$$

The system throughput $S$ is derived from the effective channel utilization. It is defined as the ratio of time taken to successfully transmit payload bits to the sum of the packet transmission time $T$ and the idle slot time $E[I]$,

$$
S=P_{s} \frac{\gamma T}{T+E[I]}
$$

where $T$ is measured in slot times,

$$
T=T_{\text {Header }}+T_{\text {Payload }}+\text { SIFS }+ \text { ACK }+ \text { DIFS }
$$

and $\gamma$ is the fraction of $T$ used for payload transmission,

$$
\gamma=\frac{T_{\text {Payload }}}{T} .
$$

In addition, the uplink throughput $S_{\mathrm{wu}}$ and the downlink throughput $S_{\text {ap }}$ are expressed as,

$$
S_{\mathrm{wu}}=P_{s}^{\mathrm{wu}} \frac{\gamma T}{T+E[I]}
$$

and

$$
S_{\text {ap }}=P_{s}^{\text {ap }} \frac{\gamma T}{T+E[I]} .
$$

Using Equation 7, $p_{\mathrm{wu}}$ can be substituted in terms of $p_{\text {ap }}$; thus, the system throughput in Equation 9 can be evaluated as follows,

$$
S=\frac{(1+k) m \gamma p_{\mathrm{ap}}\left(1-p_{\mathrm{ap}}\right)^{m+n-1}}{\left(1+p_{\mathrm{ap}}\left(\frac{\mathrm{km}}{n}-1\right)\right)^{n}+\frac{1-T}{T}\left(1-p_{\mathrm{ap}}\right)^{m+n}} .
$$

Equation 14 corroborates that $S$ depends on $p_{\text {ap }}$ which in terms of a function $C W_{\text {ap }}$ by Equation 2. An optimum value of $C W_{\text {ap }}$ is obtained when $S$ is maximized using the following equation,

$$
\frac{d S}{d \mathrm{CW}_{\mathrm{ap}}}=\frac{d S}{d p_{\mathrm{ap}}} \frac{d p_{\mathrm{ap}}}{d \mathrm{CW}_{\mathrm{ap}}}=0 .
$$


However, Equation 2 verifies that $\frac{d p_{\mathrm{ap}}}{d \mathrm{CW}_{\mathrm{ap}}} \neq 0$ (except for the lower bound condition $\left.C W_{\text {ap }} \rightarrow \infty\right)$. Hence, the solution for Equation 15 is given by $\frac{d S}{d p_{\mathrm{ap}}}=0$; this equates to,

$$
\begin{aligned}
0= & \left(\left(1+\left(\frac{\mathrm{km}}{n}-1\right) p_{\text {ap }}\right)^{n}+\frac{1-T}{T}\left(1-p_{\text {ap }}\right)^{m+n}\right)\left(1-(m+n) p_{\text {ap }}\right) \\
& -p_{\text {ap }}\left(1-p_{\text {ap }}\right)\left(\begin{array}{l}
n\left(\frac{\mathrm{km}}{n}-1\right)\left(1+\left(\frac{\mathrm{km}}{n}-1\right) p_{\text {ap }}\right)^{n-1} \\
-\frac{1-T}{T}(m+n)\left(1-p_{\text {ap }}\right)^{m+n-1}
\end{array}\right) .
\end{aligned}
$$

Considering $m+n>n \gg 1$, the following approximation holds for small $p_{\text {ap }}$,

$\left(1-p_{\mathrm{ap}}\right)^{m+n} \approx 1-(m+n) p_{\mathrm{ap}}+\frac{(m+n)(m+n-1)}{2} p_{\mathrm{ap}}^{2}$.

Thus, Equation 16 can be evaluated to give the following solution,

$$
p_{\mathrm{ap}} \approx \frac{\sqrt{(m+n)^{2}+2 Q}-(m+n)}{Q}
$$

where

$$
\begin{aligned}
Q= & \frac{n-1}{n}(\mathrm{~km}-n)^{2} T+(T-1)(m+n)(m+n-1) \\
& +2 T(\mathrm{~km}-n)(m+n-1) .
\end{aligned}
$$

$\mathrm{Q}$ is a negative number, and so Equation 18 becomes invalid when the term under the square root becomes negative, which (after some simplification) occurs when the larger of the two conditions holds

$$
n>\max [((k+1) \sqrt{2 T}-1) m, 2(T-m)] .
$$

Now, the optimum $C W_{\text {ap }}$ can be approximated from Equation 2,

$$
\mathrm{CW}_{\mathrm{ap}}^{\mathrm{opt}} \approx \frac{2 Q}{\sqrt{(m+n)^{2}+2 Q}-(m+n)} .
$$

Further, Equations 7 and 21 can be used to approximate the optimum value of $C W_{\text {wu }}$ as,

$$
\mathrm{CW}_{\mathrm{wu}}^{\mathrm{opt}} \approx \frac{n\left(\mathrm{CW}_{\mathrm{ap}}-1\right)}{\mathrm{km}}+2 \text {. }
$$

The derived formulation is verified in Appendix A.

Our proposed scheme aims to keep the CW sizes as close as possible to the optimum values at all the time. Hence, after a successful transmission, the CW sizes of the stations remain constant; they are not reset to a minimum value as in the BEB scheme.

Figure 3 illustrates the system throughput $S$ with respect to the contention window size $C W_{\text {wu }}$ for a network with $m=15, n=60, T=30$, and $\gamma=0.56$. The latter applies to the simulation parameters of Table 1 . The figure further verifies that there exists an optimum contention window size that maximizes throughput. It demonstrates that a higher value of $k(k=2)$ increases the probability of a WU transmission by reducing the optimum $C W_{\mathrm{wu}}^{\mathrm{opt}}$ size, hence, increasing the overall uplink throughput. On the other hand, a lower value of $k(k=0.5)$ reduces the probability of a WU transmission by increasing the optimum $C W_{\mathrm{wu}}^{\mathrm{opt}}$ size. The figure also shows that the formulated optimum $C W_{\mathrm{wu}}^{\mathrm{opt}}$ (Equation 22) is almost 20\% higher than the actual $C W_{\mathrm{wu}}^{\mathrm{opt}}$ (for $k=1$ ) based on brute force evaluations of Equations 3, 7, and 14. However, the difference has a negligible effect on the throughput with an error of $0.3 \%$.

The system simulations are carried out in OPNET $16.1^{\mathrm{a}}$ software using the IEEE 802.11a standard. The simulation parameters are summarized in Table 2.

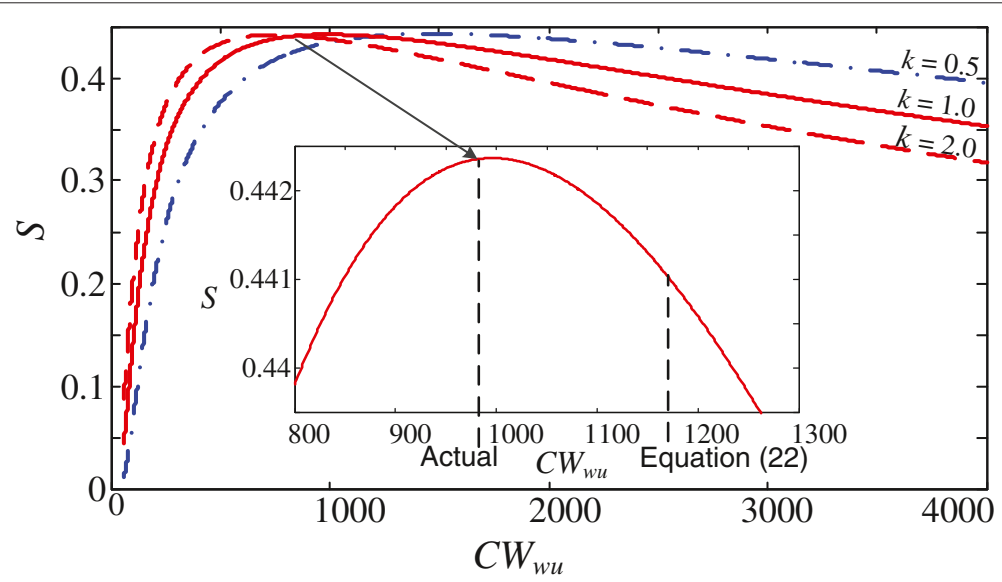

Figure 3 Normalized throughput, S vs CW wu from Equations 3, 7, and 14 with transmission priority factor $k=[0.5,1,2]$ 
Table 2 OPNET simulation parameters

\begin{tabular}{ll}
\hline Parameter & Value \\
\hline Packet size & 8,184 bits \\
\hline MAC header & 224 bits \\
\hline PHY header & $20 \mu \mathrm{s}$ \\
\hline ACK length & 134 bits/control rate + PHY header \\
\hline Data rate & $54 \mathrm{Mbps}$ \\
\hline Control rate & $6 \mathrm{Mbps}$ \\
\hline Channel bandwidth & $20 \mathrm{MHz}$ \\
\hline Slot time & $9 \mu \mathrm{s}$ \\
\hline SIFS time & $16 \mu \mathrm{s}$ \\
\hline DIFS time & $34 \mu \mathrm{s}$ \\
\hline ACK timeout & $70 \mu \mathrm{s}$ \\
\hline Packet transmission time $(T)$ & $30 \mathrm{slots}$ \\
\hline Transmission priority factor $(k)$ & 1 \\
\hline
\end{tabular}

Figure 4 compares the uplink and downlink saturation throughputs for the BEB [12] and AWA [22] schemes with the proposed TxPriority scheme having transmission priority factor $k=1$. The normalized throughput is measured as the total bits/second successfully received by each station over the channel capacity (data rate $=54 \mathrm{Mbps}$ ). The traffic load is increased by increasing the number BSSs ranging from 1 to 30; each BSS comprises of one integrated ONT-AP and four associate WUs.

As expected, lower DL throughputs are observed in comparison to UL throughputs for the BEB and AWA schemes since they are both designed for ad hoc networks. The plots show that at a high traffic load (i.e., 30 BSS), the DL throughputs for BEB and AWA schemes are 0.06 and 0.09 with the corresponding UL throughputs of 0.25 and 0.35 , respectively. These resulted in the ratio between DL

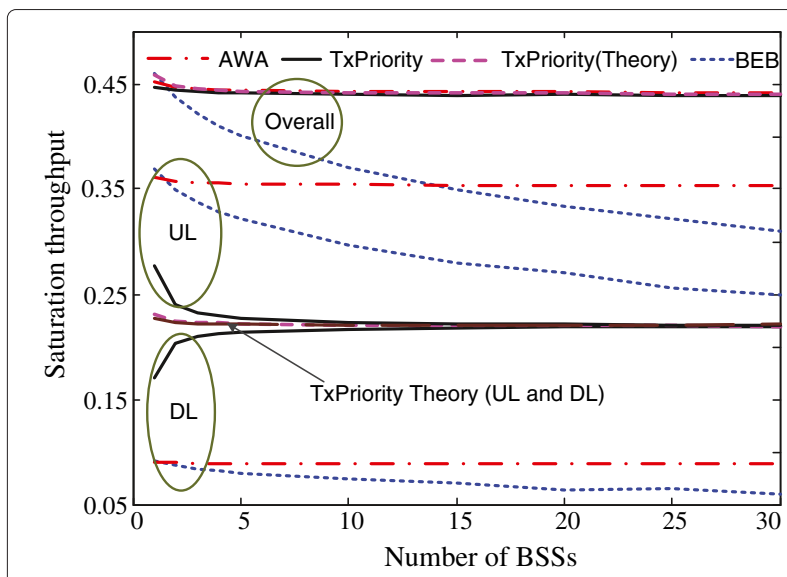

Figure 4 Normalized saturation throughput vs. number of BSSs for $B E B, A W A$, and TxPriority (simulation and theory) schemes. and UL throughputs for both schemes to be approximately 0.25 , which reflects the simulation scenario of having one AP serving every four WUs. On the other hand, our TxPriority scheme with $k=1$ has an equal DL and UL throughputs of 0.22 . An imbalance between DL and UL throughputs is noted for low BSS numbers due to the binomial approximations used in Equation 17 to derive optimum CW size (Equations 21 and 22). Apart from this imbalance, the TxPriority scheme attains throughputs close to the theoretical values derived in Equations 12 and 13 for uplink and downlink throughputs, respectively. Although the TxPriority scheme brings a balance between the uplink and downlink throughputs, it does not compromise the overall throughput. The throughput matches the theoretical results in Equation 9 and that of the AWA scheme. In both schemes, the throughput remained constant over increasing number of BSSs since the CW sizes are assigned on the number of active stations. The BEB scheme does not consider the number of contending stations; it simply doubles its CW size at every unsuccessful transmission and results in deteriorating throughput performance as the number of BSSs increases.

Larger CW sizes also result in higher media access delays. Figure 5 shows that the BEB scheme has longer delays than the AWA and TxPriority schemes. The media access delay is measured as the average time that a packet has to wait before its transmission (i.e., the backoff counter decrements to zero). Both BEB and AWA schemes have their DL access delays equal to their UL access delays. In contrast, DL delays in the TxPriority scheme are always less than UL delays because the assigned $C W_{\text {ap }}$ size is less than the $C W_{\text {wu }}$ size. Nonetheless, the average overall delay of the TxPriority scheme is equal to that of the AWA scheme and considerably better than the exponentially increasing delay of the BEB. The TxPriority scheme aims to maximize the overall throughput and achieve the desired UL/DL ratio $k$ by setting the optimum CW sizes of APs and WUs. The optimum CW sizes depend on four parameters $k, T, m$, and $n$. The priority factor $k$ is set globally in accordance to the network demand. The packet transmission time $T$ for each station is known from Equation 10. However, the values of $m$ and $n$ vary with the number of active users in the network. Hence, the scheme could be made adaptive if $m$ and $n$ were periodically estimated and each station updated their optimum $\mathrm{CW}$ sizes accordingly. The following section discusses how the estimate for $m$ can be gained with information from the GPON network.

\section{GPON network indicator}

GPON employs a time division multiplexing (TDM) for downstream $(1,480$ to $1,500 \mathrm{~nm})$ and time division multiplexing access (TDMA) for upstream $(1,260$ to 1,360 $\mathrm{nm})$ transmissions. The downstream traffic is broadcast 


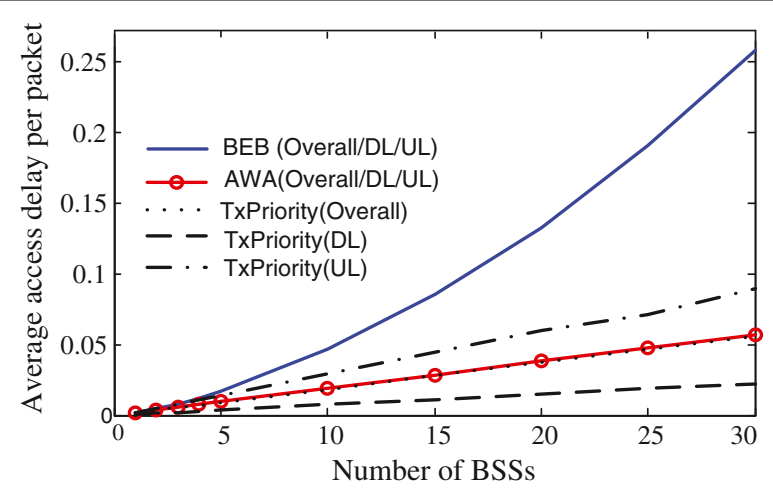

Figure 5 Media access delay per packet transmission vs. number of BSSs for BEB, AWA, and TxPriority schemes.

to all ONTs from OLT, and each frame is labeled with the address of its target ONT.

Figure 6 illustrates the GPON upstream and downstream frame format known as GPON encapsulation method (GEM) as specified in the ITU Standard G 984.3 [37]. The broadcast nature of downstream transmission allows for ONTs to retrieve network traffic information of all ONTs on the same PON. Each Port-ID within the GEM headers is unique to an ONT and is checked by all to identify their corresponding payload. Further, the upstream bandwidth mapping (US BW Map) subfield within the downstream control frame schedules upstream time slot for active ONTs. Hence, the total number of active integrated ONT-APs $m$ can be estimated at the air interface by combining the estimate of active ONTs on the upstream line and downstream line, respectively. It is assumed that the GPON network is primarily used for high speed data services, and an active ONT-AP implies that it is serving at least one associate WU. Since the estimate $m$ is only available to APs and not WUs, each

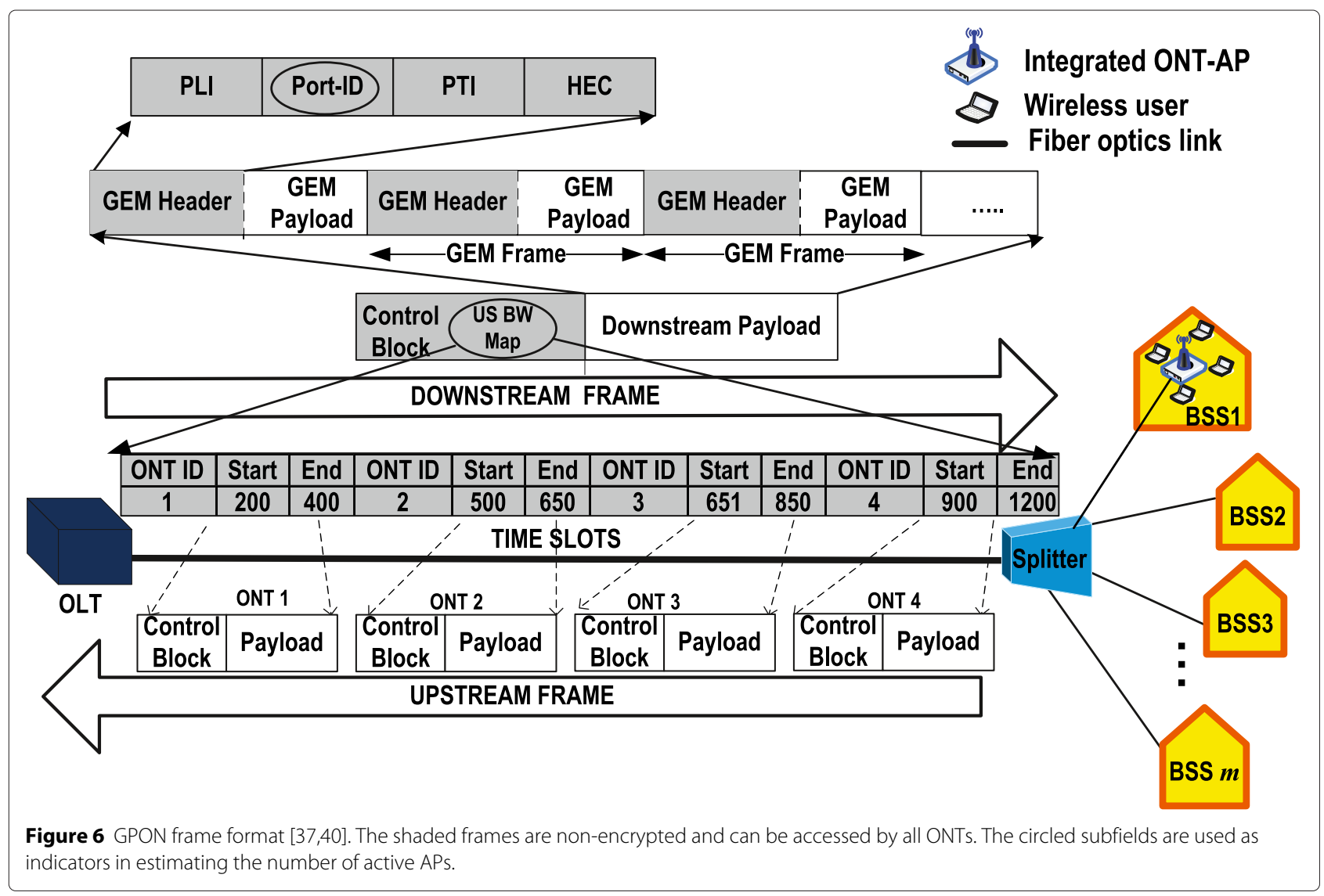


AP will periodically broadcast the information to their associate WUs.

The next section proposes a technique for estimating the number of active WUs $n$, thus, allowing each station to set optimum CW size.

\section{Adaptive backoff technique}

The value of $n$ can only be estimated by observing the activity of the channel. The probability that there is a transmission on the channel can be approximated as,

$$
\hat{P}_{\text {tr }}=\frac{B}{B+I}
$$

where $B$ is the number of times the channel is busy within an observation period (i.e., starting from the time a station first contends the channel until it completes transmission) and $I$ is the total number of idle slots.

Figure 7 illustrates an example of the estimate for station Y. Station Y with a packet to transmit has randomly chosen a backoff counter of 10. The backoff counter is decremented while the channel is sensed idle. When the backoff counter is decremented to 5 , the channel is sensed busy due to station X's transmission. As a result, the backoff counter is frozen at 5 until the transmission is complete (i.e., station X completes transmission) and the channel is sensed idle. Station Y waits a further DIFS period before decrementing the backoff counter to 4 . Subsequently, the backoff counter is decremented until it reaches zero and station $\mathrm{Y}$ transmits. As such, the value of $B$ observed by station $\mathrm{Y}$ is 2 (i.e., one time busy due to station X's transmission and the second due to its own transmission) and the value of $I$ is observed to be 9 . Hence, the $\hat{P}_{\text {tr }}$ is evaluated to be $2 / 11$. The stochastic process of selecting the backoff counter makes the estimate $\hat{P}_{\text {tr }}$ prone to error when measured over a single observation time. To reduce the error, the estimate is averaged over ten observation periods and is given by,

$$
\bar{P}_{\mathrm{tr}}=\frac{\sum_{i=1}^{10} B_{i}}{\sum_{i=1}^{10} B_{i}+\sum_{i=1}^{10} I_{i}} .
$$

Figure 8 compares the $\bar{P}_{\text {tr }}$ with the actual value of $P_{t r}$ from Equation 1 for a scenario of $m=15$ and $n=60$. A standard deviation error of $4.4 \%$ is noted.

The raw estimate $\hat{n}$ is approximated by employing firstorder binomial approximation into Equation 1,

$$
P_{\mathrm{tr}}=n p_{\mathrm{wu}}+m p_{\mathrm{ap}}-m n p_{\mathrm{ap}} p_{\mathrm{wu}}
$$

and substituting using (2) and (3),

$P_{\mathrm{tr}}=\frac{2 n}{\mathrm{CW}_{\mathrm{wu}}+1}+\frac{2 m}{\mathrm{CW}_{\mathrm{ap}}+1}-\frac{4 m n}{\left(\mathrm{CW}_{\mathrm{wu}}+1\right)\left(\mathrm{CW}_{\mathrm{ap}}+1\right)}$.

The algorithm starts here. At time $(t)$, the smooth estimate $\bar{P}_{\text {tr }}$ from Equation 24 is applied to Equation 26 to estimate the number of users, $\hat{n}$, (Equation 27).

$$
\hat{n}(t)=\frac{\left.\left.\left(\overline{\mathrm{CW}}_{\mathrm{wu}}+1\right)\left(\left(\overline{\mathrm{CW}}_{\mathrm{ap}}\right)+1\right) \bar{P}_{\mathrm{tr}}(t)\right)-2 m\right)}{2\left(\overline{\mathrm{CW}}_{\mathrm{ap}}+1-2 m\right)} .
$$

Furthermore, a smoother behaving estimate $\bar{n}$ is obtained by exploiting a first-order recursive filter of $\hat{n}$,

$$
\bar{n}(t)=\alpha \bar{n}(t-1)+(1-\alpha) \hat{n}(t)
$$

where $\alpha$ is a smoothing factor. A value of $\alpha=0.8$ is chosen since it has been shown in literature $[18,22]$ and [19] as a good compromise between accuracy and precision.

The updated $\bar{n}(t)$ evaluates the new estimate of the $\overline{\mathrm{CW}}_{\mathrm{ap}}(t)$ and $\overline{\mathrm{CW}}_{\mathrm{wu}}(t)$, formulated in Equations 21 and 22 as follows,

$$
\begin{aligned}
& \overline{\mathrm{CW}}_{\mathrm{ap}}(t) \approx \frac{2 \bar{Q}}{\sqrt{(m+\bar{n}(t))^{2}+2 \bar{Q}}-(m+\bar{n}(t))} \\
& \overline{\mathrm{CW}}_{\mathrm{wu}}(t) \approx \frac{\bar{n}(t)\left(\overline{\mathrm{CW}}_{\mathrm{ap}}(t)-1\right)}{\mathrm{km}}+2
\end{aligned}
$$

where the $\bar{Q}$ is calculated from Equation 19 using $\bar{n}(t)$. The process (Equation 24 to Equation 30) is then repeated with the new $\overline{\mathrm{CW}}(t)$ 's for the next time period.

$\overline{\mathrm{CW}}_{v w u}=\overline{\mathrm{CW}}_{\mathrm{wu}}(t)$

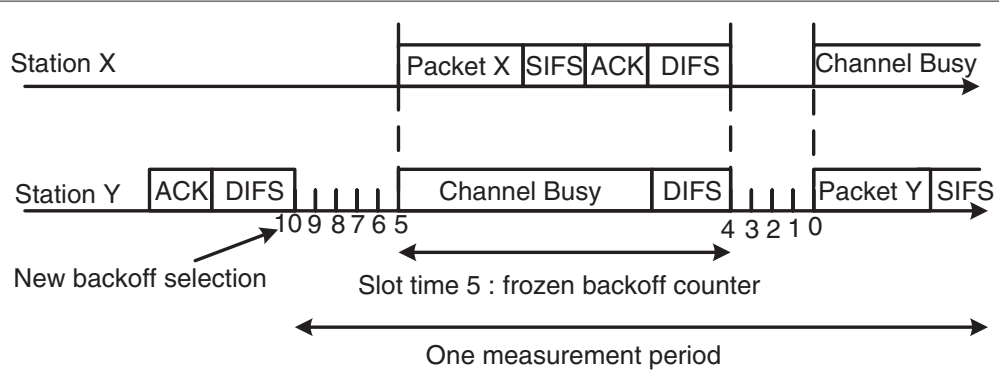

Figure 7 Estimating $\hat{P}_{\text {tr }}$ over one observation period as measured by station $\mathrm{Y}$. 


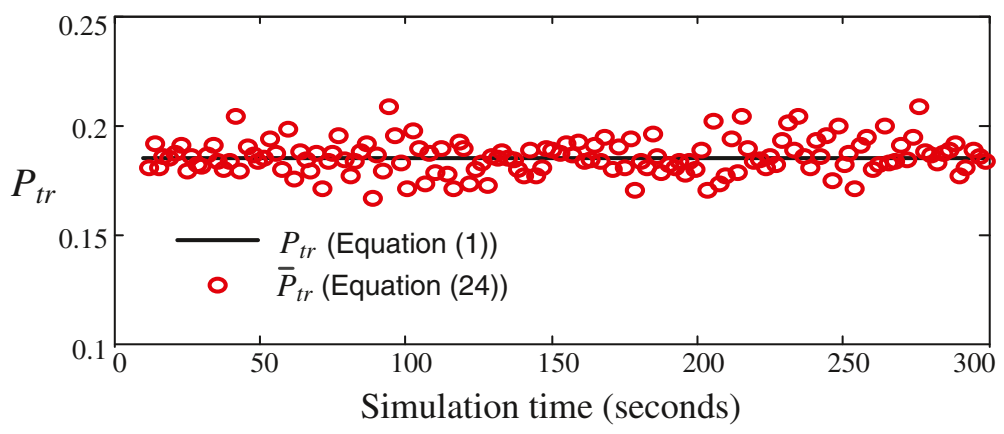

Figure 8 Average $P_{\text {tr }}$ values as measured by one user (Equation 24) compared to theoretical $P_{t r}$ values (Equation 1).

and,

$$
\overline{\mathrm{CW}}_{\mathrm{ap}}=\overline{\mathrm{CW}}_{\mathrm{ap}}(t) \text {. }
$$

As it stands, the proposed adaptive technique does not converge to the optimum CW due to $\hat{n}$ not reaching the correct value. Figure 9 shows the effect of adaption on one station with all the other stations using the correct value of $n=60$ and inhibiting their correction algorithms. When the adapting station starts off with an initial estimate above the correct value, $(\bar{n}(t=0)=70)$, it further diverges until it reaches a stable value of $\bar{n}(t \rightarrow \infty)=200$, far above the optimum value and resulting in a $\mathrm{CW}$ much larger than optimum. Similarly when starting with a lower estimate $(\bar{n}(t=0)=50)$, the estimate drops to close on zero, forcing a small CW. Both situations cause the measured $P_{\operatorname{tr}}$ to change and affect the estimates of the other stations. In particular, the low CW causes the adapting station to hog the traffic which gives an impression to other stations that a higher number of users are accessing the channel; this is indicated by their estimates going over the actual value or $n=60$.

\section{The convergence function}

In this section, a new convergence function is added into the computation of $\mathrm{CW}(t)$. The convergence function acts

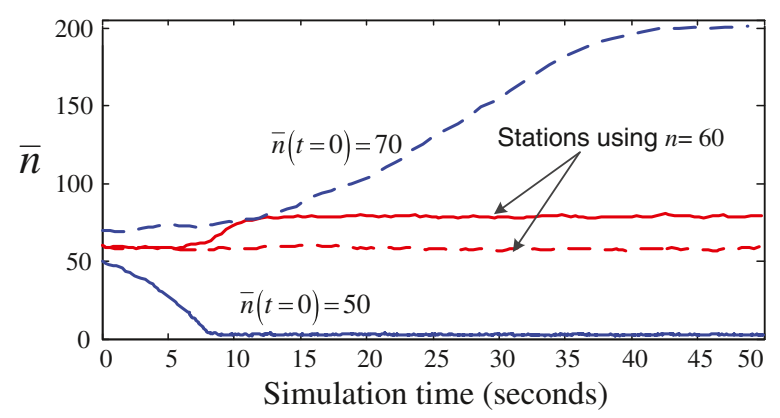

Figure 9 The convergence of $\bar{n}$ for one station. Other stations use ideal $(n=60)$. Initial condition: below $\bar{n}(t=0)=50$, solid; above $\bar{n}(t=0)=70$, dashed. as a correcting factor in estimating the $\hat{n}$. In what follows, an analysis is carried out to show that the convergence function for an ad hoc network described in [22] is not appropriate for an infrastructure multiple AP scenario.

Equations 31 and 32 are replaced by:

$$
\overline{\mathrm{CW}}_{\mathrm{wu}}=c(\bar{n}) \overline{\mathrm{CW}}_{\mathrm{wu}}(t)
$$

$$
\overline{\mathrm{CW}}_{\mathrm{ap}}=c(\bar{n}) \overline{\mathrm{CW}}_{\mathrm{ap}}(t)
$$

where the convergence function,

$$
c(\bar{n})=\left(1+\frac{h}{\sqrt{\bar{n}(t)}}\right) .
$$

Under conditions $C W_{\text {ap }} \gg 1$ and $C W_{\text {wu }} \gg 1$, Equation 27 can be simplified and rewritten as,

$$
\hat{n}(t)=\frac{\left(\overline{\mathrm{CW}}_{\mathrm{wu}}\right)\left(\left(\overline{\mathrm{CW}}_{\mathrm{ap}}\right) P_{\mathrm{tr}}-2 m\right)}{2\left(\overline{\mathrm{CW}}_{\mathrm{ap}}-2 m\right)} .
$$

The (:) represents the estimate by the adapting station while $P_{\mathrm{tr}}$ is the actual value dominated by other stations which we assume operating close to the optimum CW size in which case the assumption that they all have the same $p_{\text {ap }}$ or $p_{\text {wu }}$ is justified. Substituting $P_{\operatorname{tr}}$ in Equation 36 with Equation 26 gives

$$
\begin{aligned}
\hat{n}=\frac{\overline{\mathrm{CW}}_{\mathrm{ap}} \overline{\mathrm{CW}}_{\mathrm{wu}}}{\overline{\mathrm{CW}}_{\mathrm{ap}}-2 m}\left(\frac{n}{\mathrm{CW}_{\mathrm{wu}}}+\frac{m}{\mathrm{CW}_{\mathrm{ap}}}-\frac{2 m n}{\mathrm{CW}_{\mathrm{ap}} \mathrm{CW}_{\mathrm{wu}}}\right. \\
\left.-\frac{m}{\mathrm{CW}_{\mathrm{ap}}}\right) .
\end{aligned}
$$

where $n$ and $m$ are the actual number of wireless users and access points, all (except for the adapting station) operating with contention windows of $C W_{\mathrm{wu}}$ and $C W_{\mathrm{ap}}$, respectively. The convergence improves with the positive integer convergence factor, $h$, in Equation 35 . However, Figure 10 indicates that the system throughput gradually decreases as the convergence factor increases. This observation is justified by Equation 33 to 35; increasing the value of $h$ will further increase the $\mathrm{CW}$ size beyond 


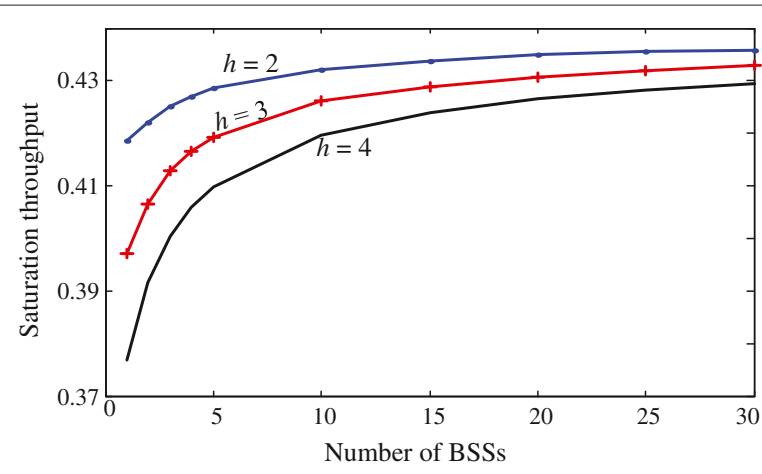

Figure 10 Effect of the convergence factor to the system throughput as the number of BSSs increases.

the optimum value and result in throughput degradation. Therefore, lower values of $h$ are preferred.

An analysis is carried out to investigate the compatibility of the convergence function with the formulated CW size. Figure 11 plots $\hat{n}-\bar{n}$ against $\bar{n}$ as it deviates from the actual value of $n$ for different sizes of $m$ and varying values of $h$. To achieve effective convergence, $\hat{n}-\bar{n}>0$ (i.e., $\hat{n}>\bar{n}$ ) is required when $\bar{n}<n$ and $\hat{n}-\bar{n}<0$ (i.e., $\hat{n}<\bar{n}$ ) is required when $\bar{n}>n$. The convergence region is graphically represented by the shaded areas in Figure 11; while the non-shaded areas represent divergence. A stable value for $\bar{n}$ exists when the curve crosses the zero correction line with a negative slope. The steeper the curve the stronger the stability. For example, with no correction factor, $c(\bar{n})=1$, the curve is almost completely in the divergence region. If $\bar{n}<n$, then $\bar{n}$ will keep reducing until it gets to 0 ; or if it is greater that $n$, it will keep increasing until the curve re-enters the convergence region at a stable but incorrect value of $\bar{n}$. In Figure $11 \mathrm{~b}$, the incorrect value is $\bar{n}=200$, way in excess of the actual value of $n=60$ but in agreement with the simulations of Figure 9 which use the same values of $n, m$, and $k$.
Figure 11a illustrates the results for $m=5$ having $n=$ 20 . The line $h=1$ falls outside the shaded region and will not drive $\bar{n}$ toward the actual value of $n$. Nonetheless, the lines $h=2, h=3$, and $h=4$ all fall within the shaded region and will allow convergence, $h=2$ being the smallest results in the least throughput degradation and is considered to be the optimum choice. However, in Figure11b,c, for $m=15$ and $m=30$, both with the same $n / m=4$ ratio, the optimum value of $h$ increases to $h=3$ and $h=4$ respectively, indicating that the proposed scheme has to adaptively choose the optimum $h$ for a particular size of $m$, thus, increasing its complexity. This is because the convergence equation defined in Equation 35 does not consider the number of APs $(m)$. Hence, a modified convergence function $c(m, \bar{n})$ is proposed,

$$
c(m, \bar{n})=\left(1+\frac{h+2 \log _{10} m}{\sqrt{\bar{n}(t)}}\right) .
$$

Figure $12 \mathrm{a}, \mathrm{c}$ shows the results of the modified convergence function for the more extreme cases $m=5$ and $m=30$. All lines now fall within the shaded regions for
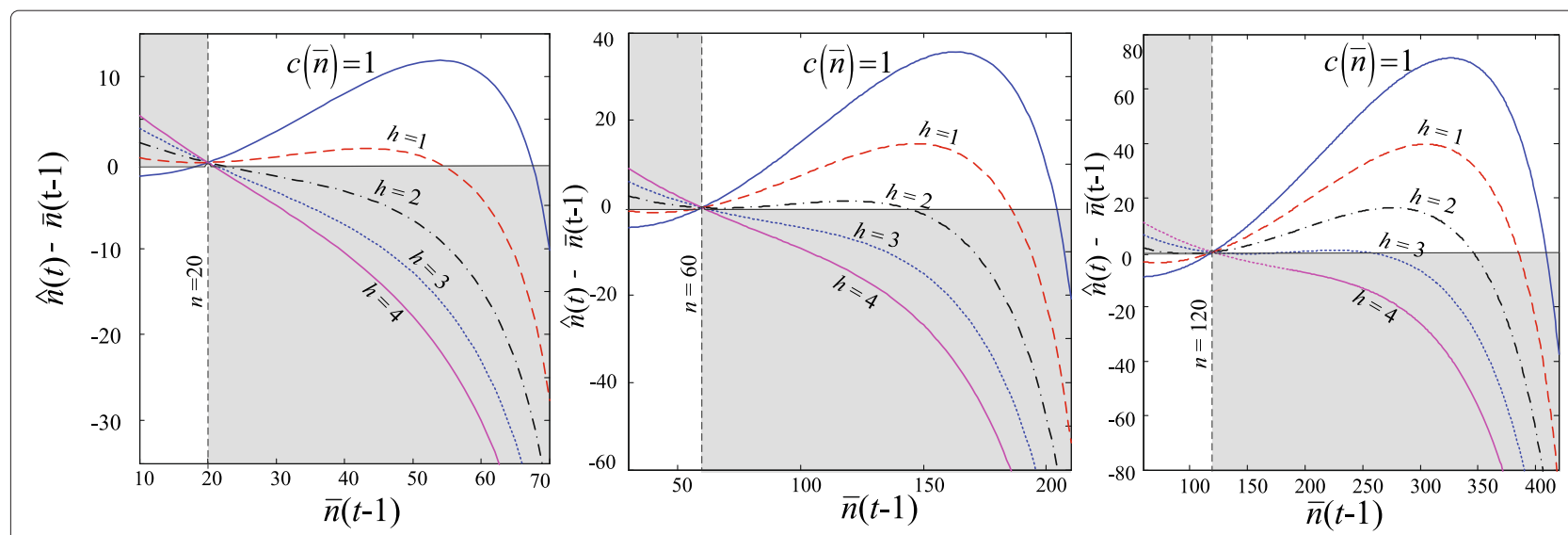

Figure 11 The change in $\bar{n}$ per iteration vs. $\bar{n}$ for different values of $h,\left(c(\bar{n})=\left(1+\frac{h}{\sqrt{n}}\right)\right)$ 


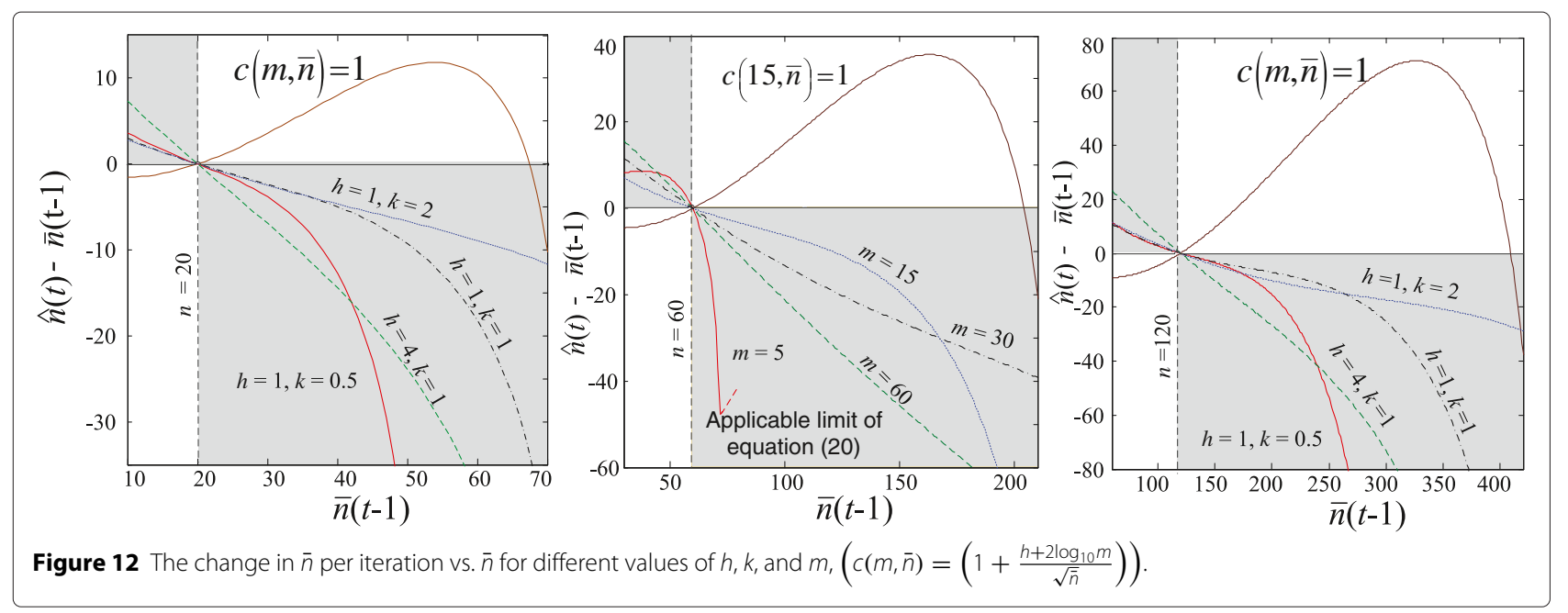

$k$ between 0.5 and 2 . As a result, the constant $h=1$ can be used as the optimum choice irrespective of the size of $m$. Figure $12 \mathrm{~b}$ shows the curves stay in the stable zones as the ratio $n / m$ changes while holding the number of users constant at $n=60$. The truncation of the $m=5$ curve for large $\bar{n}$ is due to the invalidity condition (Equation 20). The maximum number of APs simulated was 60, but since the curves appear to be moving into more stable regions of the graph as $m$ increases, the upper value of $m$ for stability is likely to be much higher than this. However, the situation is quite different for $n$, which is limited by condition (Equation 20). For large $m$, the maximum value of $n$ is set by the ratio $n / m<((k+1) \sqrt{2 T}-1)$. Using typical values of $k=1$ and $T=30, n<14.4 m$ contending users, a figure that is unlikely to be exceeded in a GPON residential environment. For small $m$, the $n / m$ limit improves (increases) since the second term of Equation 20 now dominates.

Finally, the new convergence function can be incorporated into Equations 29 and 30 to give the $\mathrm{CW}$ sizes as follows,

$\overline{\mathrm{CW}}_{\mathrm{ap}}=\left(1+\frac{1+2 \log _{10} m}{\sqrt{\bar{n}}}\right) \frac{2 \bar{Q}}{\sqrt{(m+\bar{n})^{2}+2 \bar{Q}}-(m+\bar{n})}$

and,

$$
\overline{\mathrm{CW}}_{\mathrm{wu}}=\left(1+\frac{1+2 \log _{10} m}{\sqrt{\bar{n}}}\right)\left(\frac{\bar{n}\left(\overline{\mathrm{CW}}_{\mathrm{ap}}-1\right)}{\mathrm{km}}+2\right) .
$$

Figure 13 illustrates the $\overline{\mathrm{CW}}_{\text {wu }}$ of one particular station for simulation scenarios of $5 \mathrm{BSSs}, 15 \mathrm{BSSs}$, and $30 \mathrm{BSSs}$ contending the channel. It is observed that all $\overline{\mathrm{CW}}_{\text {wu }}$ converge within $15 \mathrm{~s}$. The $\overline{\mathrm{CW}}_{\text {wu }}$ for 5 BSSs converge almost instantaneously after the simulation starts.
The converged values of $\overline{\mathrm{CW}}_{\mathrm{wu}}$ are larger than the $\mathrm{CW}_{\mathrm{wu}}^{\mathrm{opt}}$ values by, $34 \%, 28 \%$, and $25 \%$ for 5 BSSs, 15 BSSs, and 30 BSSs, respectively; a combined effect of the convergence function and other approximations. The error percentages decrease as the size of BSSs increases due to the fact that the defined convergence function (Equation 38) is a decreasing one. However, as seen in Figure 14, the overall system throughput of the proposed adaptive scheme, called the adaptive TxPriority (ATxPriority), only degrades by $3 \%$ compared to the TxPriority scheme.

Further, Figure 15 plots the cumulative distribution function (cdf) of all the WU stations' $\overline{\mathrm{CW}}_{\mathrm{wu}}$ for three network sizes after the networks have converged. In common, the slopes of the obtained cumulative distribution functions are steep, corroborating the small standard deviations, $\sigma_{\overline{\mathrm{CW}}_{\mathrm{wu}}}$, of : $2.3 \%, 1.1 \%$, and $1.3 \%$ (relative to the measured means $\mu \overline{\mathrm{CW}}_{\mathrm{wu}}$ ) for 5 BSSs, 15 BSSs, and 30

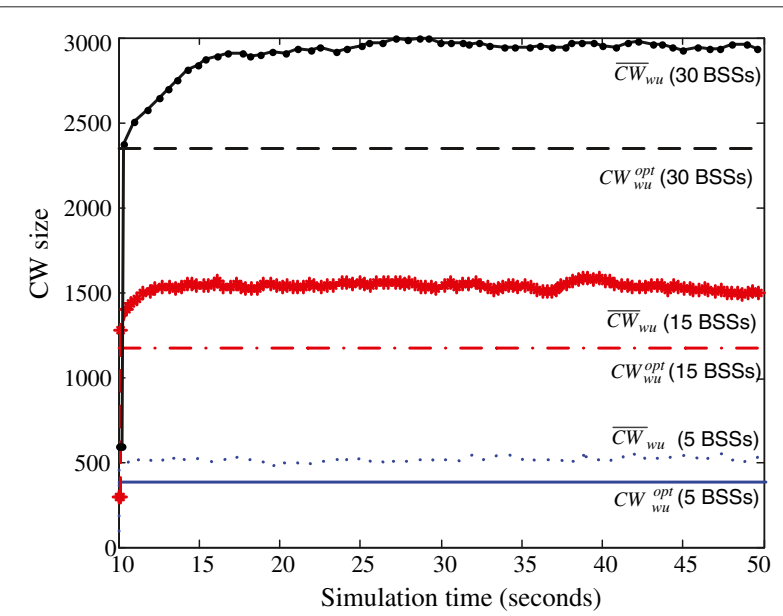

Figure 13 The convergence of $\overline{C W}_{w u}$ toward $\mathrm{CW}_{w u}^{\text {opt }}$ of one station for three sizes of BSS. 


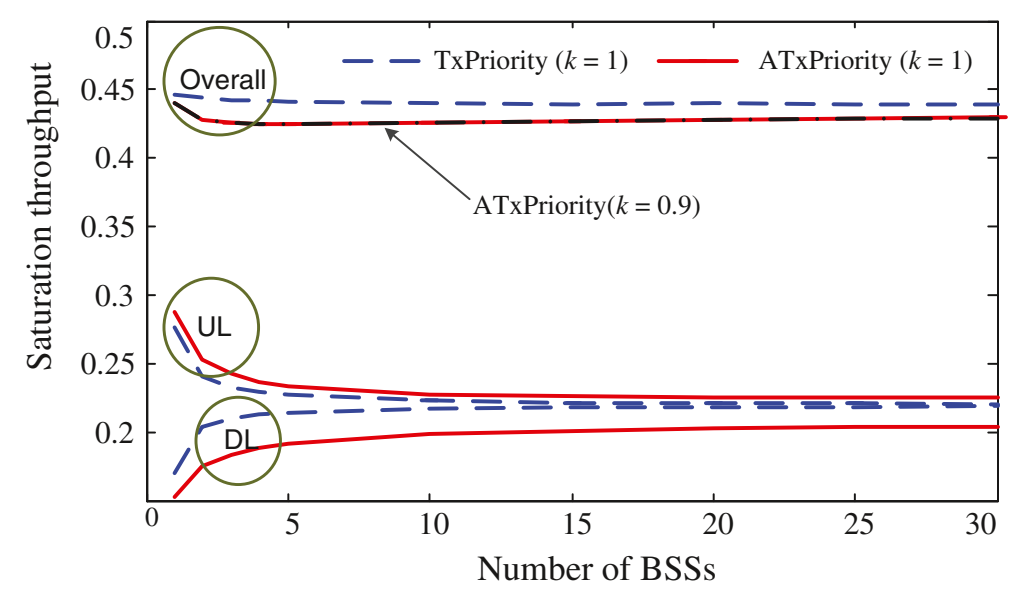

Figure 14 Comparison of normalized saturation throughput vs. number of BSSs between TxPriority and ATxPriority schemes.

BSSs, respectively. This leads to the conclusion that most WU stations within a network have an equal chance of transmission, thus, offering comparable fairness among all WUs.

Figure 16 compares the measured transmission priority factor $k_{\text {mea }}(\mathrm{UL} / \mathrm{DL})$ of the ATxPriority scheme to the TxPriority scheme. A $10 \%$ reduction is observed. This is due to a higher degradation of the downlink throughput experienced by the ATxPriority scheme than its uplink throughput, as shown in Figure 14. Equation 7 shows that the balance between the downlink throughput and the uplink throughput can be recovered by reducing the value of $k$. As illustrated in Figure 16, a value of $k=0.9$ brings the measured transmission priority factor $k_{\text {mea }}=$ 1. Nonetheless, the overall throughput of the ATxPriority remains unaltered (Figure 14, $k=0.9$ ).

\section{Conclusions}

The paper proposes a transmission priority scheme with an adaptive backoff technique to enhance the WLAN performance of a Fi-Wi (GPON-WLAN) hybrid.The scheme introduces a transmission priority factor $k$ to control the UL/DL fairness. The optimum CW sizes for $\mathrm{AP}$ and WU are derived. Performance evaluations show that the scheme is comparable to the adaptive window algorithm (AWA) scheme in terms of overall throughput and delay. Nonetheless, it outperforms the legacy BEB scheme with $40 \%$ overall throughput improvement and a maximum $80 \%$ of delay reduction. The adaptive backoff technique is introduced. This requires estimates of the number of APs $(m)$ and the number of WUs $(n)$. The first is directly estimated from GPON frame information, and the second is estimated by measuring the activity on the WLAN channel. However, the studied behavior of $\bar{n}$ (estimate of $n$ ) indicates that it does not converge to the correct value. Thus, a new convergence function, $c(m, \bar{n})$, is added. Simulations show its robustness for practical values of $k, m$, and $n$; but there is a small $3 \%$ reduction in overall throughput and a slight offset in $k$. The latter can be corrected by pre-compensation. The scheme shows

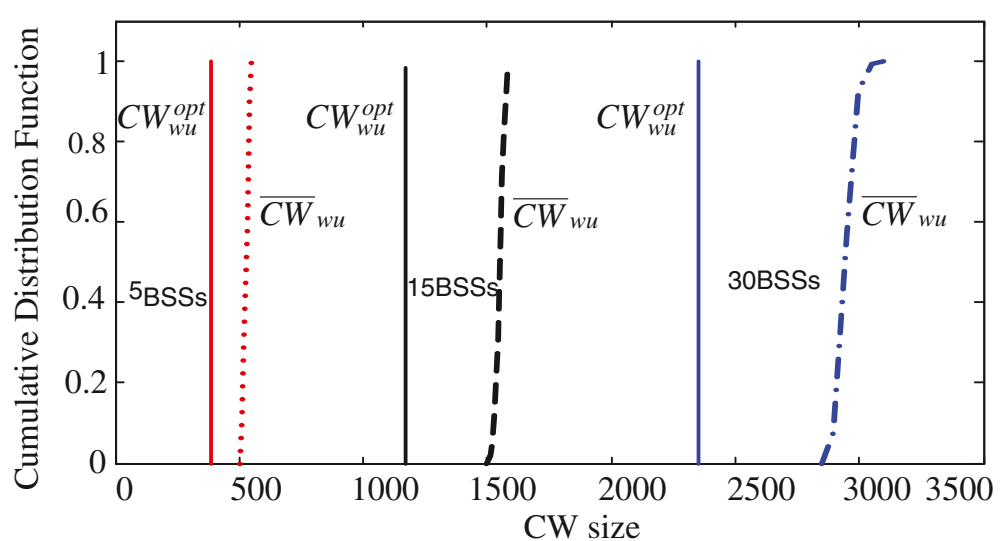

Figure 15 The cumulative distribution function of $\overline{\mathrm{CW}}_{\text {wu }}$ of contending WUs for three sizes of BSS. 


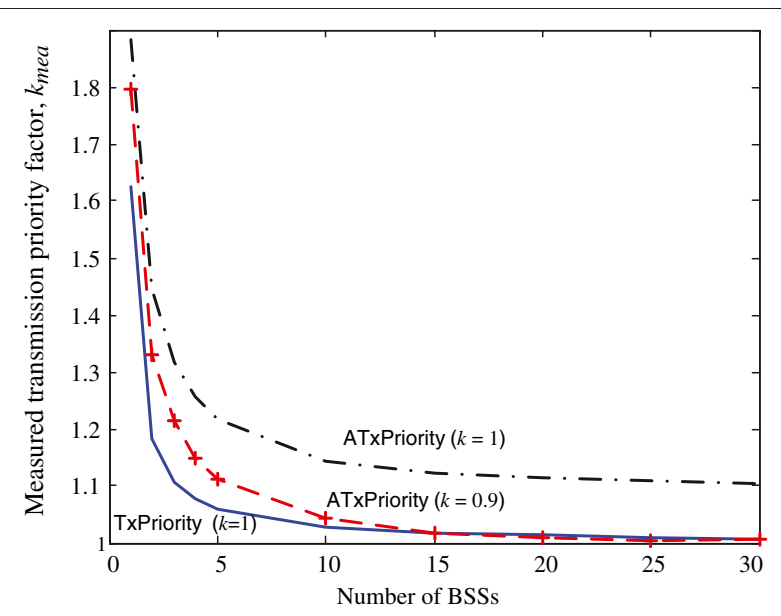

Figure 16 Comparison of the measured transmission ratio factor between TxPriority and ATxPriority schemes for different sizes of BSSs.

uniform convergence among all WUs with a measured standard deviation of their CW sizes being less than $1.5 \%$. It is worth noting that the proposed algorithm is compatible with the whole range of WLAN standards because it is aimed at improving the legacy $B E B$ technique in the DCF protocol adopted by all 802.11 family members $[24,38]$. The scheme improves the integration of fiberwireless networks as it ensures each station in the BSS is given a fair transmission opportunity so that the huge bandwidth capacity provided by the GPON (backhaul) can be fully utilized. Our future work will further improve the scheme by utilizing additional information from GPON and removing some of the constraints, for example, allowing neighboring GPON clusters and non-closed spectrum.

\section{Endnote}

${ }^{a}$ OPNET software was chosen over other simulation packages because of their vast library of built-in functions.

\section{Appendix A : validation of the formulated optimum CW size}

The following formulation extension is carried out to validate the derived formulations with the previous published work in [22]. The formulation can be extended to an $\mathrm{ad}$ hoc network by letting $p_{\mathrm{ap}}=p_{\mathrm{wu}}=p$, which implies both stations are having equal chance of transmitting. As such, Equation 7 becomes

$$
k=\frac{n}{m}
$$

Then, Equation 18 becomes

$$
\begin{aligned}
p & =\frac{\sqrt{(m+n)^{2}+2(T-1)(m+n)(m+n-1)}-(m+n)}{(T-1)(m+n)(m+n-1)} \\
& \approx \frac{\sqrt{2}}{(m+n) \sqrt{T}} .
\end{aligned}
$$

Let the total number of stations, $m+n$, equals to $N$ and so the optimum $C W$ can be approximated from Equation 3 as,

$$
\mathrm{CW}^{\mathrm{opt}} \approx \sqrt{2 T} N
$$

The above derived equation is identical to the optimum contention window size formulated in [22].

\section{Competing interests}

The authors declare that they have no competing interests.

\section{Acknowledgements}

We acknowledge the support from OPNET Technologies Inc. for providing us the educational version of OPNET 16.1 software. We also acknowledge Prof. Giuseppe Bianchi, for his kindness in providing us further information on proving the stability of an estimate.

Received: 4 November 2014 Accepted: 8 March 2015

Published online: 03 April 2015

\section{References}

1. M Milosavljevic, P Kourtessis, A Gliwan, JM Senior, in Proc. ICTON 2009, paper Tu.A5.4. Advanced PON topologies with wireless connectivity (Azores, Portugal, 2009)

2. BO Obele, MH Kang, in Proc. IEEE NGMAST. Fixed mobile convergence: a self-aware QOS architecture for converging WiMAX and GEPON access networks (Wales, UK, 2008), pp. 411-418

3. G Shen, RS Tucker, CJ Chae, Fixed mobile convergence architectures for broadband access: integration of EPON and WIMAX. IEEE Commun. Mag. 45(8), 44-50 (2007)

4. N Ghazisaidi, M Martin, M Reisslein, VMP: A MAC protocol for EPON-based video-dominated FiWi access networks. IEEE Trans. Broadcasting. 58.3(2012), 440-453 (2012)

5. J Wang, K Wu, S Li, C Qiao, Performance modeling and analysis of multi-path routing in integrated fiber-wireless networks, (San Diego, USA 2010), pp. 311-315

6. M Maier, M Herzog, STARGATE: The next evolutionary step toward unleashing the potential of WDM EPONs. IEEE Commun. Mag. 45(5), 50-56 (2007)

7. F Aurzada, M Lèvesque, M Maier, FiWi access networks based on next-generation PON and gigabit-class WLAN technologies: a capacity and delay analysis. IEEE Trans. Netw. Mag. 22(4), 1176-1189 (2014)

8. S Bindhaiq, ASM Supa, N Zulkifli, AB Mohammad, RQ Shaddad, MA Elmagzoub, A Faisal, Recent development on time and wavelength-division multiplexed passive optical network (TWDM-PON) for next-generation passive optical network stage 2 (NG-PON2). Optical Switching Netw. 15(2015), 53-66 (2014)

9. F Beltràn, Fibre-to-the-home, high-speed and national broadband plans: tales from down under. Telecommun. Policy. 38(8), 715-729 (2014)

10. M Maier, N Ghazisaidi, M Reisslein, in Proc. AccessNets. The audacity of fiber-wireless (FiWi) networks (Hong Kong, China, 2009), pp. 16-35

11. HT Win, AK Pathan, On the issues and challenges of fiber-wireless (Fi-Wi) networks. J. Eng. (2013). [Online] Available: http://dx.doi.org/10.1155/ 2013/645745

12. IEEE 802.11. standard for wireless LAN medium access control (MAC) and physical layer (PHY) specifications (2007) 
13. G Bianchi, Performance analysis of the IEEE 802.11 distributed coordination function. IEEE J. Selected Areas Commun. 18(3), 535-547 (2000)

14. N-O Song, B-J Kwak, J Song, LE Miller, in Proc. IEEE VTC-Spring Enhancement of IEEE 802.11 distributed coordination function with exponential increase exponential decrease backoff algorithm, vol. 4 (Jeju, South Korea, 2003), pp. 2775-2778

15. V Bharghavan, A Demers, S Shenker, L Zhang, in Proc. SIGCOMM94. MAWAW: A media access protocol for wireless LANs (London, England, 1994), pp. 212-225

16. WHW Hassan, H King, M Faulkner, in Proc. 23rd IEEE International Symposium on Personal, Indoor and Mobile Radio Communications (PIMRC 2012). Modified backoff technique in fiber-wireless networks (Sydney, Australia, 2012), pp. 431-435

17. I Mouhamad, S Alouf, in NETWORKING 2006 (Networking Technologies, Services, and Protocols; Performance of Computer and Communication Networks; Mobile and Wireless Communications Systems). Design and analysis of an adaptive backoff algorithm for IEEE 802.11 DCF mechanism (Springer Berlin, Heidelberg, 2006), pp. 184-196

18. F Cali, M Conti, E Gregori, IEEE 802.11 protocol: design and performance evaluation of an adaptive backoff mechanism. IEEE J. Selected Areas Commun. 18(9), 1774-1786 (2000)

19. F Cali, M Conti, E Gregori, Dynamic tuning of the IEEE 802.11 protocol to achieve a theoretical throughput limit. IEEE/ACM Trans. Netw. 8(6), 785-790 (2000)

20. P Chatzimisions, V Vitasas, A Boucouvalas, M Tsoulfa, Achieving performance enhancement in IEEE 802.11 WLANs by using DIDD backoff mechanism. Int. J. Commun. Syst. 20, 23-41 (2007)

21. K Hong, S Lee, K Kim, Y Kim, Channel condition based contention window adaptation in IEEE 802.11 WLANs. IEEE Trans. Commun. 60(2), 469-478 (2012)

22. G Bianchi, L Fratta, M Oliveri, in Proc. IEEE Personal, Indoor and Mobile Radio Communications (PIMRC'96). Performance evaluation and enhancement of the CSMA/CA MAC protocol for 802.11 wireless LANs (Taipei, Taiwan, 1996), pp. 392-396

23. G Cena, L Seno, A Valenzano, C Zunino, On the performance of IEEE 802.11e wireless infrastructures for soft-real-time industrial applications. IEEE Trans. Industrial Inform. 6(3), 425-437 (2010)

24. S Mangold, S Choi, P May, O Klein, G Hiertz, L Stibor, in Proc. European Wireless. IEEE 802.11e wireless LAN for quality of service, vol. 2 (Florence, Italy, 2002), pp. 32-39

25. B Luciano, M Conti, E Gregori, Runtime optimization of IEEE 802.11 wireless LANs performance. IEEE Trans. Parallel Distributed Syst. 15(1), 66-80 (2004)

26. E Lopez-Aguilera, M Heusse, Y Grunenberger, F Rousseau, A Duda, J Casademont, An asymmetric access point for solving the unfairness problem in WLANs. IEEE Trans. Mobile Comput. 7(10), 1213-1227 (2008)

27. WHW Hassan, $\mathrm{H}$ King, S Ahmed, M Faulkner, in Proc. IEEE African Continent Conference (AFRICON 2013). Modified backoff technique with priority transmission scheme in fiber-wireless networks (Mauritius, 2013)

28. M Al-Hubaishi, T Alahda, R Alsaqour, A Berqia, M Abdelhaq, O Alsaqour, Enhanced binary exponential backoff algorithm for fair channel access in the IEEE 802.11 medium access control protocol. Int. J. Commun. Syst. 27(12), 4166-4184 (2013)

29. BAHS Abeysekera, T Matsuda, T Takine, Dynamic contention window control mechanism to achieve fairness between uplink and downlink flows in IEEE 802.11 wireless LANs. IEEE Trans. Wireless Commun. 7(9), 3517-3525 (2008)

30. Y Gao, L Dai, Optimal downlink/uplink throughput allocation for IEEE 802.11 DCF networks. IEEE Wireless Commun. Lett. 2(6), 627-630 (2013)

31. WS Lim, DW Kim, YJ Suh, Achieving fairness between uplink and downlink flows in error-prone WLANs. IEEE Commun. Lett. 15(8), 822-824 (2011)

32. C Wang, Achieving per-flow and weighted fairness for uplink and downlink in IEEE 802.11 WLANs. EURASIP J. Wireless Commun. Netw. 1(2012), 1-9 (2012)

33. WS Kim, BS Kim, Y Fang, Downlink and uplink resource allocation in IEEE 802.11 wireless LANs. IEEE Trans. Vehic. Technol. 54(1), 320-327 (2005)

34. F Micò, J Villalón, A Valverde, in Wireless and Mobile Networking Conference (WMNC). Guaranteed access mode for downlink traffic over IEEE 802.11 WLANs (Toulouse, France, 2011)
35. G Min, J Hu, ME Woodward, Modeling and analysis of TXOP differentiation in infrastructure-based WLANs. ELSEVIER Comput. Netw. 55(11), 2545-2557 (2011)

36. OPNET Modeler 16.1. http:www.opnet.com

37. ITU-T Recommendation G.984.3. Gigabit-capable Passive Optical Networks (GPON): transmission convergence layer specification (March 2008)

38. D Skordoulis, Q Ni, H-H Chen, AP Stephens, C Liu, A Jamalipour, IEEE 802.11n MAC frame aggregation mechanisms for next-generation high-throughput WLANs. IEEE Wireless Commun. 15(1), 40-47 (2008)

39. J Johny, S Shashidharan, in Proc. 4th IEEE International Congress on Ultra Modern Telecommunications and Control Systems and Workshops (ICUMT). Design and simulation of a Radio Over Fiber system and its performance analysis (St. Petersburg, Russia, 2012), pp. 636-639

40. Huawei Technologies, GPON fundamentals [Online] Available: http:// www.huawei.com

\section{Submit your manuscript to a SpringerOpen ${ }^{\mathcal{O}}$ journal and benefit from:}

- Convenient online submission

- Rigorous peer review

- Immediate publication on acceptance

- Open access: articles freely available online

- High visibility within the field

- Retaining the copyright to your article

Submit your next manuscript at $>$ springeropen.com 